\title{
Comparing Simulations of Lipid Bilayers to Scattering Data: The GROMOS 43A1-S3 Force Field
}

\author{
Anthony R. Braun, ${ }^{\dagger}$ Jonathan N. Sachs, ${ }^{\dagger}$ and John F. Nagle*, \\ ${ }^{\dagger}$ Department of Biomedical Engineering, University of Minnesota, Minneapolis, Minnesota \\ ${ }^{\ddagger}$ Department of Physics, Carnegie Mellon University, Pittsburgh, Pennsylvania
}

Supporting Information

ABSTRACT: Simulations of DOPC at $T=303 \mathrm{~K}$ were performed using the united atom force field 43A1-S3 at six fixed projected areas, $A_{\mathrm{P}}=62,64,66,68,70$, and $72 \AA^{2}$, as well as a tensionless simulation that produced an average $A_{\mathrm{NPT}}=$ $65.8 \AA^{2}$. After a small undulation correction for the system size consisting of 288 lipids, results were compared to experimental data. The best, and excellent, fit to neutron scattering data occurs at an interpolated $A_{\mathrm{N}}=66.6 \AA^{2}$ and the best, but not as good, fit to the more extensive $\mathrm{X}$-ray scattering data occurs at

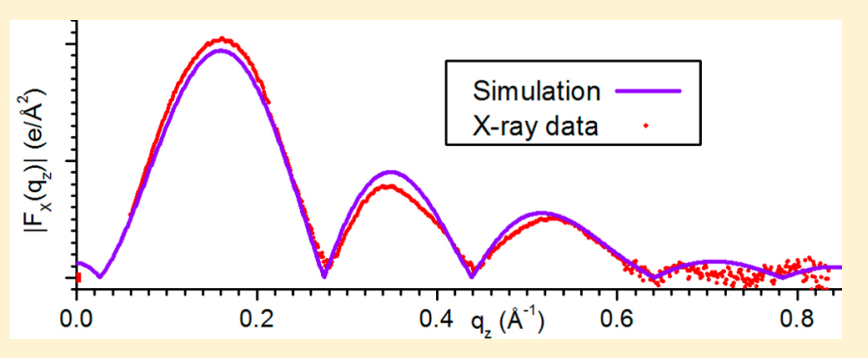
$A_{\mathrm{X}}=68.7 \AA^{2}$. The distance $\Delta D_{\mathrm{B}-\mathrm{H}}$ between the Gibbs dividing surface for water and the peak in the electron density profile agrees with scattering experiments. The calculated area compressibility $K_{\mathrm{A}}=277 \pm 10 \mathrm{mN} / \mathrm{m}$ is in excellent agreement with the micromechanical experiment. The volume per lipid $V_{\mathrm{L}}$ is smaller than volume experiments which suggests a workaround that raises all the areas by about $1.5 \%$. Although $A_{\mathrm{X}} \neq A_{\mathrm{N}} \neq A_{\mathrm{NPT}}$, this force field obtains acceptable agreement with experiment for $A_{\mathrm{L}}=67.5 \AA^{2}\left(68.5 \AA^{2}\right.$ in the workaround), which we suggest is a better DOPC result from 43A1-S3 simulations than its value from the tensionless NPT simulation. However, nonsimulation modeling obtains better simultaneous fits to both kinds of scattering data, which suggests that the force fields can still be improved.

\section{INTRODUCTION}

It is well-recognized that molecular dynamics simulations provide a level of quantitative detail unavailable to experiment. It is also well-recognized that this detail depends on the force fields used in the simulation. Accordingly, force field development and refinement for lipid bilayer simulations is ongoing. ${ }^{1-13}$ An important test of force fields is comparing simulation results to experimental scattering data. ${ }^{1-4,8,14-22}$ Many earlier tests have compared to quantities, such as area per molecule or electron density profiles, that were derived by modeling the data; in contrast, this paper compares the simulation directly to the data, bypassing any intermediate modeling. The first specific goal of this paper is to show how to carry out a recently proposed refinement of these tests for lipid bilayers. ${ }^{23}$ The main proposed refinement determines both the area $A_{\mathrm{X}}$ at which the simulation best agrees with the X-ray data and the area $A_{\mathrm{N}}$ at which it best agrees with the neutron data and asks how these areas agree with each other as well as with the area $A_{\mathrm{NPT}}$ for a tensionless simulation.

Our second goal is to critically test a particular united-atom force field 43A1-S3. ${ }^{1}$ When it was developed, this force field was tested favorably against experimental $\mathrm{X}$-ray, $\mathrm{NMR}$, and volumetric data for DLPC, DMPC, DPPC, and DOPC lipid bilayers. It was not, however, compared to neutron scattering data which has become particularly important regarding the area per lipid molecule $A_{\mathrm{L}} \cdot{ }^{18}$ Also, X-ray scattering data have since been refined from many data sets and more accurate uncertainties provided. Furthermore, only tensionless (NPT) simulations were previously performed and insight can be gained from other values of $A_{\mathrm{L}}$. We have restrained ourselves in this paper to the DOPC bilayer. This is partly because there are more extensive scattering data for DOPC than for other lipids and partly because DOPC has been a particularly hard case for other simulations. $^{23}$

\section{METHODS}

MD Simulations. The initial starting configurations were obtained from a previously simulated fluid-phase bilayer system comprised of 288 Berger et al. ${ }^{25}$ DOPC lipids and 9428 SPC waters ( 32.7 waters/lipid corresponding to fully hydrated $\left.\mathrm{DOPC}^{24}\right)$. The system was repurposed for the Chiu et al. lipid potentials, and the SPC water was replaced with SPCE. ${ }^{1}$ This system was then simulated for $100 \mathrm{~ns}$ under the isothermalisobaric (NPT) ensemble with constant pressure and temperature (1 bar and $303 \mathrm{~K}$, respectively). Pressure coupling was applied using a semi-isotropic scheme, with the $x y$-periodic box dimensions coupled, and the $z$-dimension was allowed to vary freely. Figure 1 illustrates the $A_{\mathrm{P}}$ trajectory from the NPT system showing rapid convergence to an equilibrated $A_{\mathrm{P}}=65.8$ $\AA^{2}$ that agrees with Chiu et al. ${ }^{1}$ Initial configurations for

Received: February 18, 2013

Revised: April 2, 2013

Published: April 5, 2013 


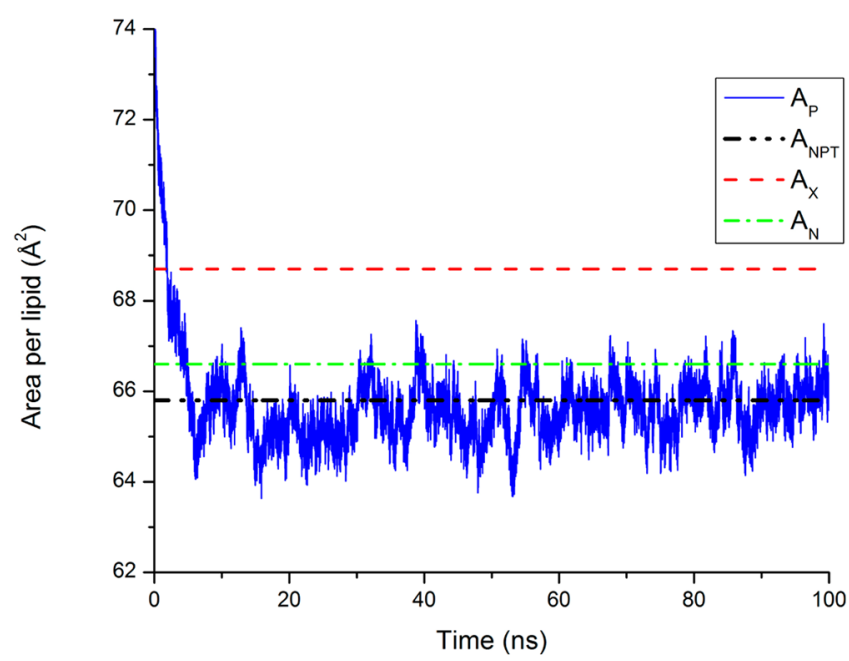

Figure 1. Time evolution of the projected area per lipid from the NPT simulation illustrating rapid convergence to an average $A_{\mathrm{P}}=65.8 \AA^{2}$. Lines for $A_{\mathrm{NPT}}$ (black), $A_{\mathrm{X}}$ (red), and $A_{\mathrm{N}}$ (green) are provided for comparison.

constant area $\left(\mathrm{NP}_{\mathrm{N}} \mathrm{AT}\right.$ ensemble) simulations were obtained from simulated frames at or near the desired projected area per lipid $\left(A_{\mathrm{P}}=62,64,66,68,70\right.$, and $\left.72 \AA^{2}\right)$ with the $x y$-box dimensions modified to achieve the specific $A_{\mathrm{P}}$. These systems were then simulated for $50 \mathrm{~ns}$ at constant area where only the $z$-dimension of the periodic cell was allowed to fluctuate. The final $20 \mathrm{~ns}$ of each simulation were used for subsequent analysis.

All systems were simulated with the GROMACS 4 program using a leapfrog algorithm to integrate the equations of motion. ${ }^{26-29}$ Each system was run at $303 \mathrm{~K}$ using a 2 fs time step and recording coordinates every 5 ps. All run parameters were obtained from Chiu et al. ${ }^{1}$ as follows. The particle mesh Ewald method ${ }^{30}$ was used for long-range electrostatics with a direct space cutoff of $1.0 \mathrm{~nm}$, Fourier spacing of $0.15 \mathrm{~nm}$, and a sixth-order interpolation. A twin-range cutoff $(1.0 / 1.6 \mathrm{~nm})$ was applied for van der Waals interactions, and the neighbor-pair list was updated every five time steps. Lipid bonds were constrained using the LINCS algorithm. ${ }^{31}$ Water bonds were constrained using the SETTLE algorithm. ${ }^{32}$ A Nosé-Hoover thermostat $^{33}$ with a time constant of 0.5 ps was used to control the ensemble temperature, while a Parrinello-Rahman barostat $^{34}$ with a time constant of 1 ps was used to keep the pressure fixed.

Determination and Comparison of Structural Profiles. Number density profiles and local area per lipid $\left(A_{\mathrm{L}}\right)$ were determined using the MDAnalysis software package ${ }^{35}$ and the surface referencing undulation correction method developed by Braun et al. ${ }^{36}$ Number density profiles were calculated for each unique united atom type ( 54 in DOPC, 3 in SPCE) with a cutoff wavenumber of $q_{0}=1.0\left[\mathrm{~nm}^{-1}\right]$ to correct for bilayer undulations. These number density profiles were then used for input into the SIMtoEXP software program ${ }^{20}$ that produces electron density profiles and their Fourier transforms, which are the X-ray form factors $F_{\mathrm{X}}\left(q_{z}\right)$, and neutron scattering length profiles and their Fourier transform, which are the neutron form factors $F_{\mathrm{N}}\left(q_{z}\right)$. SIMtoEXP imports experimental form factor data, and it provides the unknown experimental scaling factor by minimizing the $\chi^{2}$ in fitting the data to each simulation. In addition to this straightforward procedure, an alternative procedure modified the simulated volumes of the water and the lipid by multiplying the original simulated water number density profiles by one factor to obtain the experimental density of water and by multiplying the lipid number density profiles by another factor to obtain the experimental lipid volume. The latter modification means that the simulated lipid area is also multiplied by the same factor.

Simulated volumes of water, lipid, and its components were obtained using a SIMtoEXP app. ${ }^{37}$ We first defined the components to be water, choline $(\mathrm{Chol})$, phosphate $\left(\mathrm{PO}_{4}\right)$, glycerol (Gly), carbonyls (Carbs), chain methylenes $\left(\mathrm{CH}_{2}\right)$, methines $(\mathrm{CH})$, and chain terminal methyls $\left(\mathrm{CH}_{3}\right)$. We also defined the components according to the SDP model ${ }^{18}$ which combines the carbonyls and the glycerol and separates the phosphocholine into just the choline methyls and the rest. There were negligible differences in the volumes of the lipid $V_{\mathrm{L}}$ and the lipid headgroups $V_{\mathrm{H}}$ using the two different definitions of the components as shown in Tables S1 and S2 (Supporting Information).

Table 1. ${ }^{a}$

\begin{tabular}{|c|c|c|c|c|c|c|c|c|}
\hline property & experiment & & & & mulatio & & & \\
\hline$A_{\mathrm{P}}$ & & 62 & 64 & 65.79 & 66 & 68 & 70 & 72 \\
\hline$A_{\mathrm{L}}$ & & 62.2 & 64.15 & 65.89 & 66.15 & 68.14 & 70.13 & 72.12 \\
\hline$A_{\mathrm{LM}}$ & & 63.1 & 65.0 & 66.8 & 67.0 & 69.0 & 70.9 & 72.8 \\
\hline$V_{\mathrm{W}}$ & 30.0 & 30.1 & 30.1 & 30.1 & 30.1 & 30.1 & 30.1 & 30.1 \\
\hline$V_{\mathrm{L}}$ & 1303 & 1283 & 1285 & 1286 & 1286 & 1288 & 1289 & 1289 \\
\hline$V_{\mathrm{H}}$ & $319-331$ & 329 & 330 & 331 & 329 & 330 & 330 & 331 \\
\hline$r$ & $1.8-2.1$ & 2.0 & 2.01 & 2.03 & 1.96 & 1.94 & 1.96 & 1.98 \\
\hline$\Delta D_{\mathrm{H}-\mathrm{C}}$ & & 4.1 & 4.0 & 4.0 & 4.0 & 4.0 & 4.0 & 4.0 \\
\hline$\Delta D_{\mathrm{P}-\mathrm{H}}$ & & 0.9 & 0.7 & 0.6 & 0.7 & 0.7 & 0.6 & 0.5 \\
\hline$\Delta D_{\mathrm{B}-\mathrm{H}}$ & $1.0-1.7$ & 1.2 & 1.2 & 1 & 1.1 & 0.9 & 0.8 & 0.7 \\
\hline
\end{tabular}

${ }^{a} A_{\mathrm{P}}$ is the box area divided by half the number of lipids, $A_{\mathrm{L}}$ takes into account the larger local area due to undulations, and $A_{\mathrm{LM}}$ is modified as described in the Methods and Results. The volume subscripts are $\mathrm{L}$ for total lipid volume, $\mathrm{C}$ for hydrocarbon chains beginning at the second carbon, and $\mathrm{H}$ for the headgroup volume consisting of the carbonyls, glycerol, and phosphocholine. $\Delta D_{\mathrm{H}-\mathrm{C}}$ is the difference between half the head-head distance $D_{\mathrm{HH}}$ in the electron density profile and the Gibbs dividing surface $D_{\mathrm{C}}$ of the hydrocarbon region. $\Delta D_{\mathrm{P}-\mathrm{H}}$ and $\Delta D_{\mathrm{B}-\mathrm{H}}$ are the differences between the mean position of the phosphate $(\mathrm{P})$ and the Gibbs dividing surface for the water (B), respectively, and $D_{\mathrm{HH}} / 2$. Units for Areas $A$, volumes $V$, and distances $D$ are in the appropriate powers of $\AA$, and the ratio $r$ of terminal methyl to methylene volumes is dimensionless. NPT simulation gave $A_{\mathrm{P}}=65.79$; other results were from NPAT simulations. Values have been rounded to the values displayed. 
Experimental Data and SDP Modeling. A composite experimental X-ray scattering data set for $F_{\mathrm{X}}\left(q_{z}\right)$ was obtained as an average using seven sets of data from oriented stacks of bilayers and three sets of data from unilamellar vesicles. ${ }^{18,38-42}$ Neutron scattering data $F_{\mathrm{N}}\left(q_{z}\right)$ in $\mathrm{D}_{2} \mathrm{O}$ were obtained from Kučerka et al. ${ }^{42}$ These were the data that were directly compared to the simulation. Although the primary thrust of this paper did not employ interpretive modeling of these data, informative modeling from these data sets was done using the SDP analysis. ${ }^{18}$ This analysis requires estimates for various constraints such as the ratios of the volumes of the component groups in the headgroup and in the tails and for the widths of the headgroup methyl distribution, the double bond distributions, and the width of the Gibbs dividing surface for the total hydrocarbon core. As these quantities do not vary significantly with area in simulations, the simulated values provided estimates $^{18}$ and we have updated those estimates using the 43A1-S3 results. Lists of the constraints are given in Table S3 (Supporting Information). As the density of X-ray data points is greater than that for neutron data, the neutron data were weighted more heavily in the fitting procedure so as to give roughly equal values for the average (i.e., reduced) $\chi^{2}$ of X-ray and neutron data.

\section{RESULTS}

Table 1 shows that the local area per lipid $A_{\mathrm{L}}$ is only about $0.1-$ $0.2 \AA^{2}$ larger than the projected area per lipid $A_{\mathrm{P}}$ of the simulation box. Although the number of lipids in the simulation is larger than most current simulations, the simulated system is still small enough to suppress most long wavelength undulations. The small, but systematic, decrease in the difference between $A_{\mathrm{L}}$ and $A_{\mathrm{P}}$ as area is increased is consistent with increased surface tension that further suppresses undulations. The volume of SPCE water in the simulations is $0.1 \AA^{3}$ greater than experiment. Table 1 also shows that the volume $V_{\mathrm{L}}$ of DOPC gradually increases with increasing $A_{\mathrm{L}}$, as would be expected because the hydrocarbon chains become more disordered. $V_{\mathrm{L}}$ also is consistently smaller than experiment. The ratio $r$ of terminal methyl to methylene volume agrees well with the acceptable range of experimental values. ${ }^{43-45}$ Volumes of all components are compiled in Tables S1 and S2 (Supporting Information).

Figure 2 shows electron density profiles for the range of fixed $A_{\mathrm{P}}$ listed in Table 1 . As expected, the bilayer becomes systematically thinner as $A_{\mathrm{P}}$ is increased and this induces the reciprocal effect of spreading the X-ray form factors $F_{\mathrm{X}}\left(q_{z}\right)$ to larger $q_{z}$, as shown in Figure 3. The most objective comparison with experimental data is with the experimental form factors. ${ }^{14,15,22}$

Figures 3 and 4 compare, respectively, the X-ray $F_{X}\left(q_{z}\right)$ and the neutron $F_{\mathrm{N}}\left(q_{z}\right)$ scattering data. The simulations agree better with the experimental data for $A$ near the middle of the simulated range, as is most clearly seen in Figure 4. However, for visual clarity in Figures 3 and 4, only one unknown scale factor for the experimental data could be chosen, whereas the scale factors for best agreement with each simulation are slightly different. Figures 3 and 4 also show results for the SDP model.

The comparison of simulation with experiment is better quantified in Figure 5 which shows reduced $\chi^{2}$ values for which the experimental scale factor was optimized separately for each simulated area. Quadratic interpolation to the minimal $\chi^{2}$ gives $A_{\mathrm{X}}=68.7 \AA^{2}$ for best agreement of the simulation with X-ray

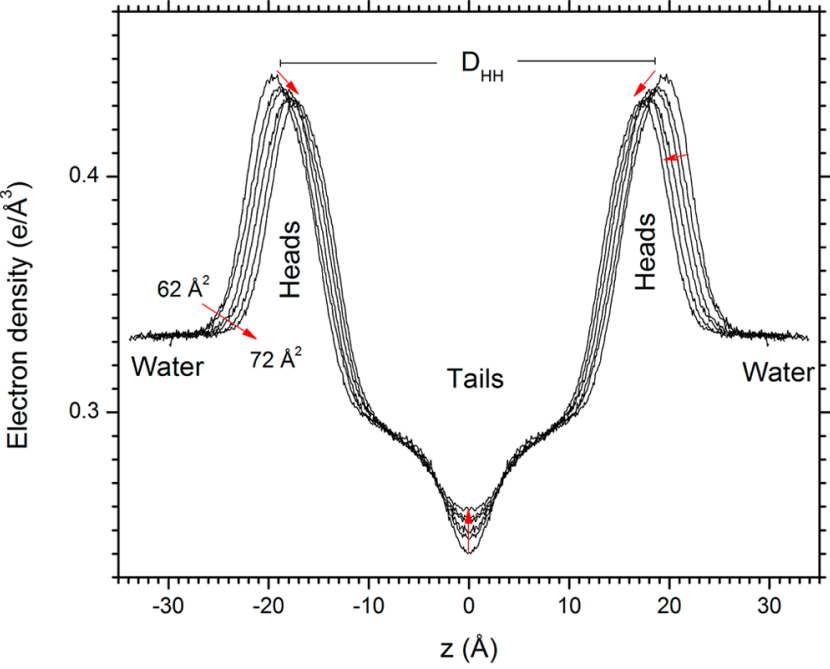

Figure 2. Simulated electron densities versus distance $z$ from the center of the bilayer for the range of projected areas. The arrows point from the smallest $A_{\mathrm{P}}=62 \AA^{2}$ to the largest $A_{\mathrm{P}}=72 \AA^{2} . D_{\mathrm{HH}}$ defines the head-head bilayer thickness.

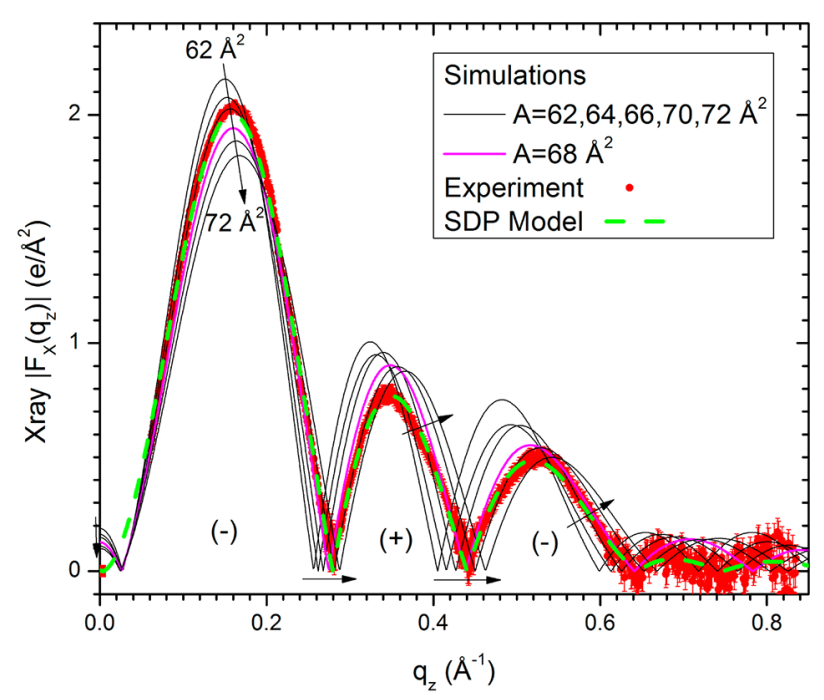

Figure 3. Simulated, experimental, and SDP model X-ray form factors I $F_{\mathrm{X}}\left(q_{z}\right) \mid$ versus $q_{z}$ in reciprocal space. The arrows indicate the progression from the smallest simulated projected area $A_{\mathrm{P}}=62 \AA^{2}$ to the largest $A_{\mathrm{P}}=72 \AA^{2}$ with the best fit to experiment shown in bold magenta. Scaled experimental data with uncertainties include the volumetric datum at $q=0$. Signs of $F_{\mathrm{X}}\left(q_{z}\right)$ are indicated by $(-)$ and $(+)$. Negative values of experimental $\left|F_{\mathrm{X}}\left(q_{z}\right)\right|$ propagate from experimental uncertainty when measured intensities are close to zero.

data and $A_{\mathrm{N}}=66.6 \AA^{2}$ for best agreement with neutron data. Figure 5 also shows results at the area $A_{\mathrm{NPT}}=65.9 \AA^{2}$ from the tensionless NPT simulation.

There is a disagreement of the simulated $F_{\mathrm{X}}\left(q_{z}\right)$ with the experimental point at $q_{z}=0$ in Figure 3 that is related to the volumetric values in Table 1 , and this leads to a possible modification in how the simulation is treated. There is a fundamental relation ${ }^{46}$

$$
A_{\mathrm{L}} F_{\mathrm{X}}(0)=2\left(n_{\mathrm{L}}-\rho_{\mathrm{W}} V_{\mathrm{L}}\right)
$$

where $n_{\mathrm{L}}=434$ is the number of electrons in DOPC, $V_{\mathrm{L}}$ is the volume of DOPC given in Table 1 , and $\rho_{\mathrm{W}}=0.333 \mathrm{e} / \AA^{3}$ is the electron density of water at $303 \mathrm{~K}$. Equation 1 gives $F_{\mathrm{X}}(0)=0$ 


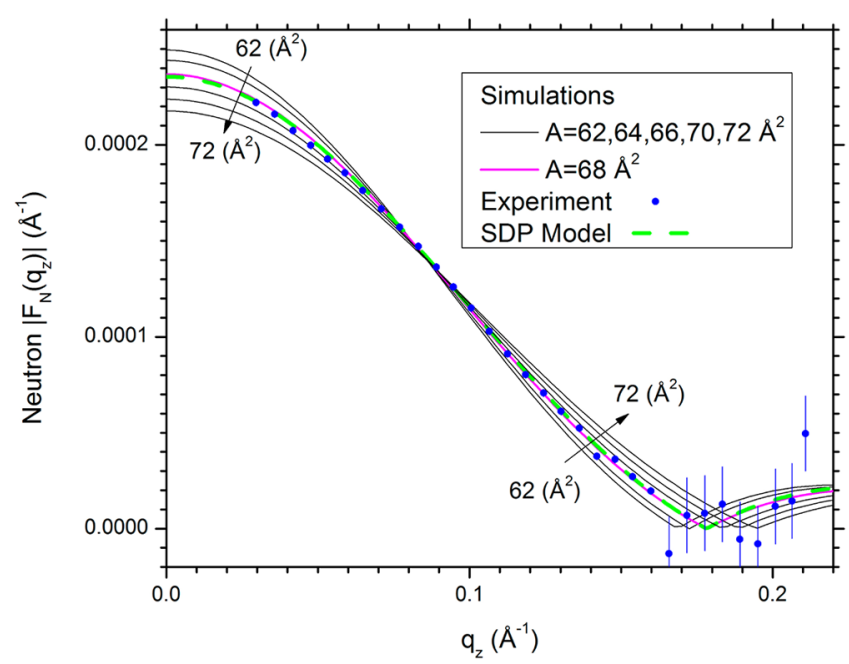

Figure 4. Simulated, experimental, and SDP model neutron form factors $F_{\mathrm{N}}\left(q_{z}\right)$ versus $q_{z}$ in reciprocal space. The arrows indicate the progression from the smallest simulated projected area $A_{\mathrm{P}}=62 \AA^{2}$ to the largest $A_{\mathrm{P}}=72 \AA^{2}$ with the best fit to experiment shown in bold magenta. Estimated uncertainties for $q_{z}<0.16 \AA^{-1}$ are about the size of the data symbols.

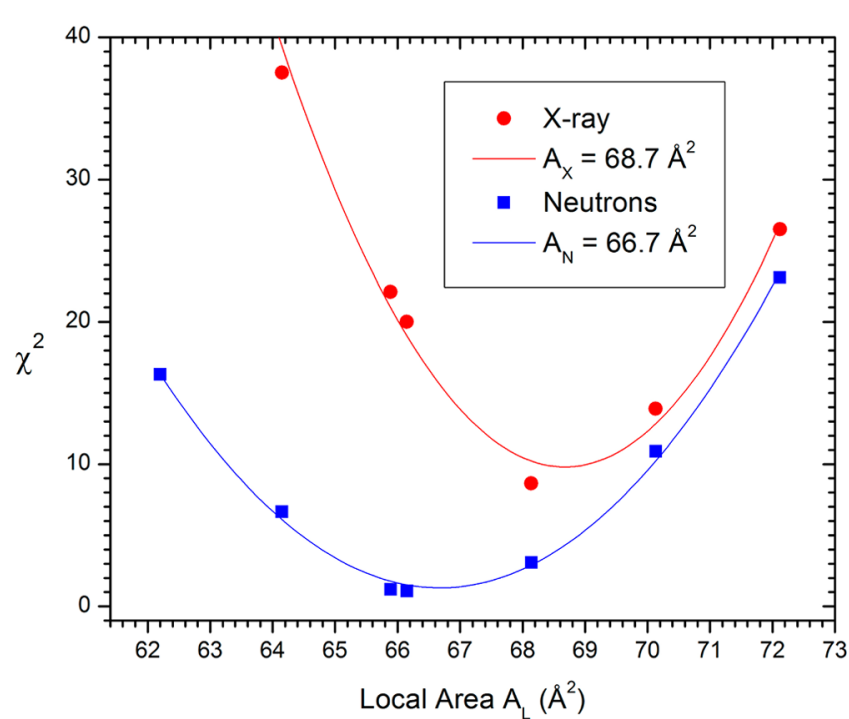

Figure 5. Symbols show values of $\chi^{2}$ from fitting simulations with local areas $A_{\mathrm{L}}$ to X-ray and neutron data, and lines show quadratic fits from which interpolated best areas $A_{\mathrm{X}}=68.7 \AA^{2}$ and $A_{\mathrm{N}}=66.7 \AA^{2}$ are obtained.

independently of the value of $A_{\mathrm{L}}$. The simulations give positive values of $F_{\mathrm{X}}(0)$ because (a) $\rho_{\mathrm{W}}$ is slightly too small due to a slightly too large $V_{\mathrm{W}}$ shown in Table 1 and (b) more significantly, the simulated $V_{\mathrm{L}}$ is also too small. These are fairly minor flaws inherent in simulations, and they invite a workaround to improve agreement with experiment, as follows. The number densities from the simulations may be multiplied by factors to give the experimental values of $V_{\mathrm{W}}$ and $V_{\mathrm{L}}$. Although one could contemplate different factors for the headgroup components than for the chain components, we have here applied the same factor for all the lipid components. Because the component distributions retain their same shape along the $z$-direction, there is no modification of volume along the $z$-direction, only in lateral directions, so lipid area should be multiplied by the same factor as the lipid volume, and all areas increase. Table 1 shows the values of the modified $A_{\mathrm{LM}}$. Figure 6 shows the results of this modification compared to the $\chi^{2}$

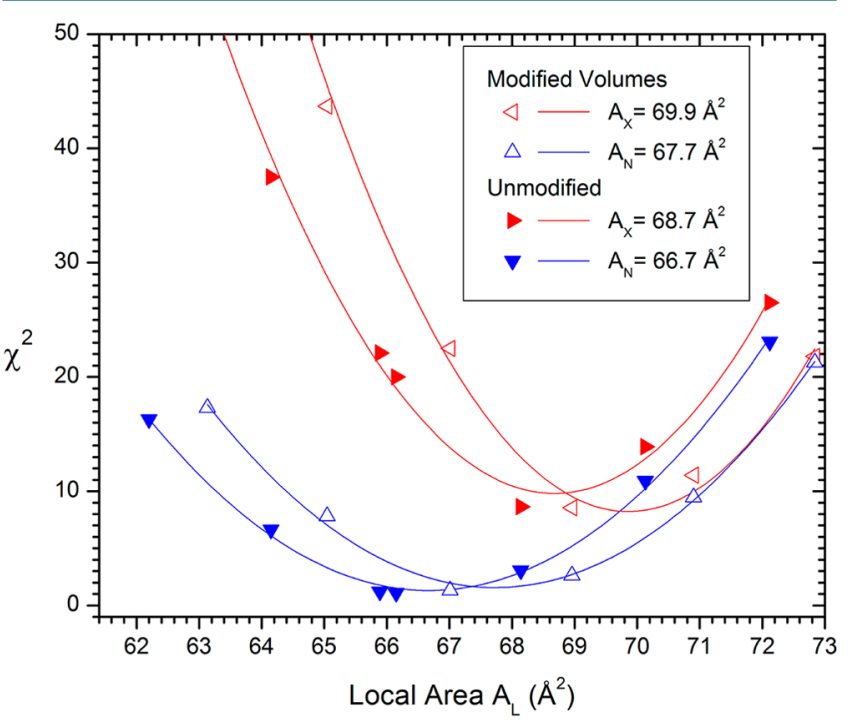

Figure 6. Same format as Figure 5 with added values for the modified volume workaround described in the text.

results repeated from Figure 5. Furthermore, the minimal value of $\chi^{2}$ improves substantially for the $\mathrm{X}$-ray, but not the neutron, comparison. Moreover, this workaround increases the difference between $A_{\mathrm{X}}$ and $A_{\mathrm{N}}$ from 2.0 to $2.2 \AA^{2}$.

Figures 3 and 4 also show results obtained from SDP modeling of the experimental data. These results agree much better with the experimental X-ray data and somewhat better with the neutron data than any of the simulations, as shown quantitatively in Table 2 .

Another property of general interest is the area compressibility modulus, defined as $K_{\mathrm{A}}=A(\partial \gamma / \partial A)_{T}=(\partial \gamma / \partial(\ln A))_{T}$. The simulations provide a value of the surface tension $\gamma$ for each simulated area $A_{\mathrm{L}}$. The simulated numerical value, $K_{\mathrm{A}}=$ $277 \pm 10 \mathrm{dyn} / \mathrm{cm}$, is obtained from the slope in the plot in Figure 7 . We also obtain $K_{\mathrm{A}}=321 \pm 37 \mathrm{dyn} / \mathrm{cm}$ using the

Table 2. ${ }^{a}$

$\begin{array}{llcl}\text { property } & \text { basic } & \text { modified } & \text { SDP } \\ A_{\mathrm{NPT}} & 65.9 & 66.7 & 67.6 \\ \chi_{\mathrm{X}+\mathrm{N}}^{2} & 11.7 & 13.3 & 1.17 \\ A_{\mathrm{N}} & 66.7 & 67.7 & \mathrm{~N} / \mathrm{A} \\ \chi_{\mathrm{N}}^{2} & 1.31 & 1.57 & 1.13 \\ A_{\mathrm{X}} & 68.7 & 69.9 & \mathrm{~N} / \mathrm{A} \\ \chi_{\mathrm{X}}^{2} & 9.81 & 8.22 & 1.21 \\ V_{\mathrm{L}} & 1287 & 1303 & 1303 \\ V_{\mathrm{C}} & 957 & 969 & 982 \\ r & 1.96 & 1.96 & 1.97\end{array}$

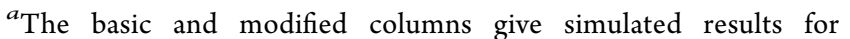
unmodified and modified simulations, respectively, where $\chi_{\mathrm{X}+\mathrm{N}}{ }^{2}$ for NPT is half the sum of the neutron and X-ray $\chi^{2}$ and $\chi_{\mathrm{N}}{ }^{2}$ and $\chi_{\mathrm{X}}{ }^{2}$ are interpolated minima, respectively, for neutron only and for X-ray only. The SDP column gives modeling results obtained from simultaneously fitting a model to both the experimental neutron and X-ray data. SDP fitting used the experimental volumes shown and ratios of other volumes consistent with the simulation as shown in Table S3 (Supporting Information). Units for all properties are in appropriate powers of $\AA$. 
fluctuation expression ${ }^{49} 2 A_{\mathrm{L}} k T / N \sigma_{\mathrm{A}}{ }^{2}$, where $\sigma_{\mathrm{A}}{ }^{2}$ is the mean square fluctuation shown in Figure 1.

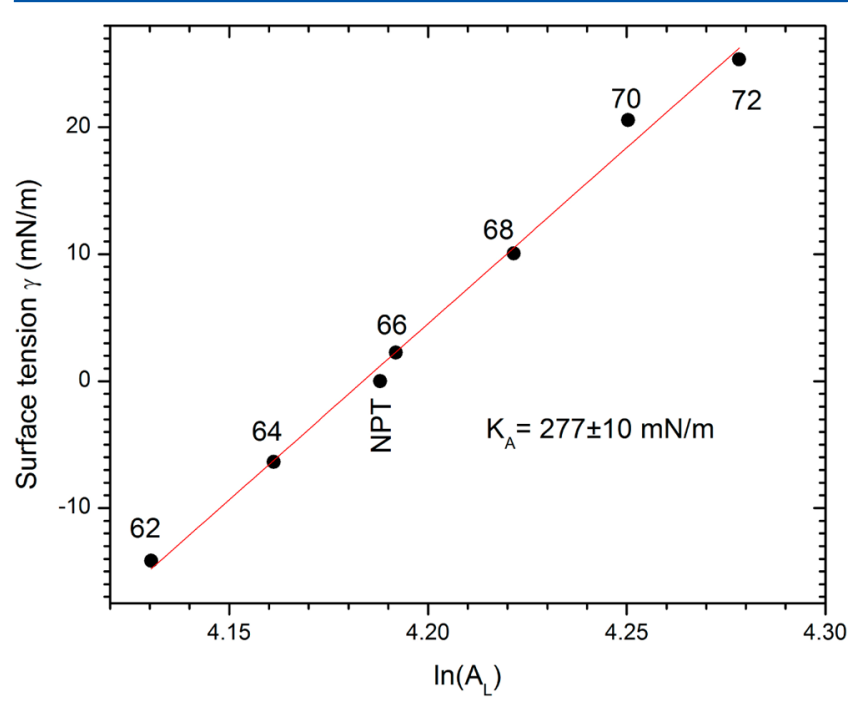

Figure 7. The slope of the simulated surface tension $\gamma$ versus the logarithm of the local area $A_{\mathrm{L}}$ provides the area compressibility modulus $K_{\mathrm{A}}$. The number next to each data point is the value of the projected area $A_{\mathrm{P}}$ in $\AA^{2}$. However, the $A_{\mathrm{L}}$ values were undulation corrected, so the slope gives the true, ${ }^{49}$ not the apparent, value for $K_{\mathrm{A}}$.

\section{DISCUSSION}

The united atom force field 43A1-S3 obtains excellent agreement with neutron scattering data, as shown qualitatively in Figure 4. Quantitatively, Table 2 shows that the reduced $\chi^{2}$ of the fit of the data to the simulated $F_{\mathrm{N}}\left(q_{z}\right)$ is close to 1 even for the NPT simulation (Figure 5) and Table 2 gives its interpolated minimum as 1.31 at $A_{\mathrm{N}}=66.7 \AA^{2}$. The $\chi^{2}$ of the fit of the X-ray data to the simulated $F_{\mathrm{X}}\left(q_{z}\right)$ is considerably poorer, as shown in Figure 5, with an interpolated minimal $\chi^{2}=9.81$ for $A_{\mathrm{X}}=68.7 \AA^{2}$. The large X-ray $\chi_{\mathrm{X}}^{2}$ reflects that the simulation has larger second and third lobes compared to the first lobe than the experimental data (Figure 3 ); a very similar $F_{\mathrm{X}}\left(q_{z}\right)$ was also reported by Chiu et al. ${ }^{1}$

One reason for the poorer agreement with the X-ray data is that they extend to much larger $q_{z}$, thereby including more structural detail and setting a greater challenge for simulations. Another reason is that the X-ray data are subject to systematic error, as seen in the slightly distorted shapes of the higher lobes of $F\left(q_{z}\right)$, though this does not account for very much of the $\chi^{2}$. A final reason is that the simulation incorrectly obtains the $F(0)$ datum because the volume per lipid $V_{\mathrm{L}}$ is too small and the volume of water $V_{\mathrm{W}}$ is too large, as shown in Table 1 , with the consequence that $F_{X}(0)$ is too large according to eq 1 . We have explored a workaround of these volumetric flaws that gives the $\chi^{2}$ results in Figure 6 with a summary of the salient results in the "modified" column in Table 2 . The workaround increases the best areas $A_{\mathrm{X}}$ and $A_{\mathrm{N}}$ by about $1.5 \%$ and it improves the fit to the X-ray data ( $\chi_{\mathrm{x}}^{2}$ decreases), as expected, but the fit to the neutron data becomes poorer.

A simulation should give $A_{\mathrm{X}}=A_{\mathrm{N}}$; that is clearly not the case with or without the volumetric workaround. However, the difference between $A_{\mathrm{X}}$ and $A_{\mathrm{N}}$ is smaller for these 43A1-S3 force field simulations than for other simulations of DOPC. ${ }^{23} \mathrm{~A}$ viable compromise value between the areas $A_{\mathrm{N}}$ and $A_{\mathrm{X}}$ obtained in this paper is an area per lipid $A_{\mathrm{L}}=67.5 \AA^{2}$ which would increase to $68.5 \AA^{2}$ for the volumetric workaround. Ideally, a simulation should also give $A_{\mathrm{L}}=A_{\mathrm{NPT}}$, but it has been argued that this is asking too much of the water potentials. ${ }^{23}$ Instead, one should accept a nonzero value of the surface tension $\gamma$ as another, quite different, workaround for flaws in the water interfacial force fields. ${ }^{17,22}$ However, for simulators who insist on only doing NPT simulations, the most appropriate number to compare is the simulated $\chi_{\mathrm{X}+\mathrm{N}}{ }^{2}(11.7$ in the basic column in Table 2) minus the experimental $\chi^{2}$ which is no larger than SDP $\chi_{\mathrm{X}+\mathrm{N}}^{2}(1.17$ in the SDP column in Table 2$)$.

It has been emphasized ${ }^{18,23}$ that the neutron $F_{\mathrm{N}}\left(q_{z}\right)$ data are most strongly sensitive to the total (Luzzati) bilayer thickness $D_{\mathrm{B}}$, so there is a best value of $D_{\mathrm{B}}$ for agreement with neutron data. The X-ray $F_{\mathrm{X}}\left(q_{z}\right)$ data are most sensitive to the headgroup peaks in the electron density profile characterized by the headhead thickness $D_{\mathrm{HH}}$ and there is a best value of $D_{\mathrm{HH}}$ for agreement with the $\mathrm{X}$-ray data. Therefore, for a simulation to have equal values of $A_{\mathrm{X}}$ and $A_{\mathrm{N}}$, it has to obtain the best values of both $D_{\mathrm{HH}}$ and $D_{\mathrm{B}}$ at the same $A_{\mathrm{L}}$. Of course, both thicknesses decrease as the area is increased, as tabulated in Tables S1 and S2 (Supporting Information). The important quantity is therefore the difference $\Delta D_{\mathrm{B}-\mathrm{H}}=\left(D_{\mathrm{B}}-D_{\mathrm{HH}}\right) / 2$. Most importantly for a force field to obtain $A_{\mathrm{X}}=A_{\mathrm{N}}$ is that $\Delta D_{\mathrm{B}-\mathrm{H}}$ agree with experiment for the most relevant values of $A_{\mathrm{L}}$ and the 43A1-S3 force field succeeds according to this metric, as shown in Table 1.

Other insights can be obtained from Table 1. The fact that the quantity $\Delta D_{\mathrm{H}-\mathrm{C}}=\left(D_{\mathrm{HH}}-2 D_{\mathrm{C}}\right) / 2$, where $2 D_{\mathrm{C}}$ is the hydrocarbon thickness, is constant suggests that the headgroup conformation does not change with $A_{\mathrm{L}}$. Then, the result that $\Delta D_{\mathrm{B}-\mathrm{H}}$ gradually decreases with increasing $A_{\mathrm{L}}$ is due to $D_{\mathrm{B}}$ decreasing more rapidly than $D_{\mathrm{HH}}$ which can be understood because water fills in more of the volume between the headgroups in the interfacial region, thereby bringing the Gibbs dividing surface for water closer to the hydrocarbon core. Table 1 also indicates that there is a nonzero distance $\Delta D_{\mathrm{P}-\mathrm{H}}$ between the average location of the phosphate and the peak in the electron density profile; this can be traced to the electron density of the carbonyl and glycerol groups being large enough to pull the peak of the total electron density from the phosphate significantly toward the center of the bilayer. Detailed locations of all the component groups and their volumes are provided in Tables S1 and S2 (Supporting Information).

A particularly noteworthy test of simulations is the ratio $r$ of the volume of the terminal methyls on the hydrocarbon chains to the volume of the methylenes. One of the reasons that this paper has focused on the 43A1-S3 force field rather than the older, much utilized Berger et al. force field ${ }^{25}$ is that the latter gives too large values of $r \sim 2.7$. Table 1 shows that the results for the 43A1-S3 force field agree well with the experimentally acceptable range. We note in passing that the Berger force field also provides excellent agreement with the X-ray and neutron experimental data at the same area $A_{\mathrm{L}}=67.4 \AA^{2}$.

An additional motivation for simulating many areas is to obtain the area compressibility modulus $K_{\mathrm{A}}=277 \mathrm{mN} / \mathrm{m}$, as shown in Figure 7. The value of $K_{\mathrm{A}}$ is insensitive to chain type for PC lipids, ${ }^{47}$ so as emphasized by Klauda et al., ${ }^{5} K_{\mathrm{A}}$ is a robust quantity for force field development. It may be noted that, for DPPC, CHARMM36 gave $K_{\mathrm{A}}$ somewhat smaller in the range $193-267 \mathrm{mN} / \mathrm{m},{ }^{4,5,48}$ and an undulation correction applied to simulations employing the Berger et al. force fields gave a larger value of $348 \mathrm{mN} / \mathrm{m} .{ }^{49,50}$ As mentioned at the end 
of the results, we have also applied the fluctuation method to our 43A1-S3 NPT data in Figure 1 and obtained $K_{\mathrm{A}}=321 \pm 37$ $\mathrm{mN} / \mathrm{m}$. Until recently, the accepted experimental value for DOPC was $K_{\mathrm{A}}=265 \pm 18 \mathrm{mN} / \mathrm{m},{ }^{47}$ although a recent reevaluation has suggested raising this to $\sim 300 \mathrm{mN} / \mathrm{m}^{51}$ so agreement of 43A1-S3 with the experimental $K_{\mathrm{A}}$ is excellent.

Determining the area $A_{\mathrm{L}}$ for DOPC has been especially challenging. For many years, $A_{\mathrm{L}}$ was reported to be about 72 $\AA^{2} .24,38,41,52$ However, simultaneous analysis of $\mathrm{X}$-ray and neutron scattering data, called the SDP analysis, lowered $A_{\mathrm{L}}$ to $67.4 \AA^{2}{ }^{18}$ Since the experimental data have been updated, we have performed the SDP analysis again and now estimate $A_{\mathrm{L}}$ $=67.6 \pm 0.5 \AA^{2}$ (from Tables S4 and S5 and column 5 of Table S3, Supporting Information). The result $A_{\mathrm{L}}=67.5 \AA^{2}$ that we suggest above to be the most appropriate unmodified result for the 43A1-S3 force field is in remarkably good agreement with the SDP results.

However, as is apparent from Figures 3 and 4 and quantified in Table 2, SDP modeling fits the experimental data much better than the simulations; this suggests that the 43A1-S3 simulations can be improved. We have explored directions such improvements might take using the SDP program. The SIMtoEXP program $^{20}$ provides simulated values for the parameters that are used in the SDP model. ${ }^{18}$ Inserting these values into the SDP program essentially recovers the fits obtained by the SIMtoEXP program. The SDP program was then run while constraining a subset of these values to see whether better $\chi^{2}$ can be obtained by allowing the other parameters to fit. Often, very good fits to the data can be obtained but the values of the parameters are completely unrealistic. For example, the distance between the Gibbs dividing surface for the hydrocarbon core $\left(D_{\mathrm{C}}\right)$ and the carbonyl/glycerol (CG) group becomes stereochemically too small, so this distance was constrained to its value $1.3 \AA$ obtained by the 43A1-S3 simulations and also by CHARMM simulations. We found that the fit was significantly improved when the widths of the headgroup distribution and the Gibbs dividing surface for the hydrocarbon core were allowed to increase. The fit also improved when the volume of the CG group was allowed to decrease. Detailed numerical results are given in Tables S3-S5 (Supporting Information). Hopefully, these clues may suggest modifications in the force fields, especially regarding the CG moiety which is the lipid backbone.

\section{CONCLUSIONS}

Lipid force field development and subsequent experimental validation continue to be faced with the fundamental challenge of defining appropriate metrics for thorough comparison to experiment. Ideally, a force field simulated at zero surface tension would agree with both neutron and X-ray data. As this does not happen for other force fields, ${ }^{23}$ we have devised a more refined test. By using a series of NPAT simulations, we compare the areas $A_{\mathrm{X}}$ and $A_{\mathrm{N}}$ at which the simulation best fits the X-ray and neutron data, respectively, and we suggest that $A_{\mathrm{X}}$ $=A_{\mathrm{N}}$ is a primary criterion for testing a simulation. Then, the comparison of the tensionless area $A_{\mathrm{NPT}}$ is a secondary criterion. This study has applied this refined test to the GROMOS 43A1-S3 united atom force field specifically for DOPC. Although agreement with neutron scattering data is excellent with $A_{\mathrm{N}}$ only $0.8 \AA^{2}$ greater than $A_{\mathrm{NPT}}$, agreement with the more challenging X-ray data is relatively poorer with $A_{\mathrm{X}}$ nearly $3 \AA^{2}$ greater than $A_{\mathrm{NPT}}$. Such detailed studies have not yet been performed for other force fields, but it appears that these results, while not perfect, make this force field quite competitive. Although our focus has been on validation with Xray and neutron scattering experiments, we have also tested the 43A1-S3 force field against volumetric data, where it obtains excellent values for the relative methyl and methylene volumes, though it obtains somewhat small values for the overall hydrocarbon volume. Also, the 43A1-S3 force field agrees very well with the $K_{\mathrm{A}}$ mechanical micromanipulation datum. We suggest that the type of analysis in this paper be performed for other force fields and also for other lipids.

\section{ASSOCIATED CONTENT}

\section{Supporting Information}

A detailed description of component structural parameters for each simulation and SDP model fit results. This material is available free of charge via the Internet at http://pubs.acs.org.

\section{AUTHOR INFORMATION}

\section{Corresponding Author}

*E-mail: nagle@cmu.edu. Phone: 412-268-2764.

\section{Author Contributions}

The manuscript was written through contributions of all authors. All authors have given approval to the final version of the manuscript.

\section{Notes}

The authors declare no competing financial interest.

\section{ACKNOWLEDGMENTS}

We thank Dr. Norbert Kučerka for maintaining the SIMtoEXP and SDP software and for compiling scattering data at http:// www.norbbi.com/. This research was supported through a F31 NRSA predoctoral fellowship (A.R.B.) and by the National Institute of General Medical Sciences of the National Institutes of Health under Award No. R01GM44976 (J.F.N.). The content is solely the responsibility of the authors and does not necessarily represent the official views of the National Institutes of Health. All simulations were performed at the Minnesota Supercomputing Institute (MSI).

\section{REFERENCES}

(1) Chiu, S. W.; Pandit, S. A.; Scott, H. L.; Jakobsson, E. An Improved United Atom Force Field for Simulation of Mixed Lipid Bilayers. J. Phys. Chem. B 2009, 113, 2748-2763.

(2) Poger, D.; Van Gunsteren, W. F.; Mark, A. E. A New Force Field for Simulating Phosphatidylcholine Bilayers. J. Comput. Chem. 2010, $31,1117-1125$.

(3) Poger, D.; Mark, A. E. On the Validation of Molecular Dynamics Simulations of Saturated and cis-Monounsaturated Phosphatidylcholine Lipid Bilayers: A Comparison with Experiment. J. Chem. Theory Comput. 2010, 6, 325-336.

(4) Klauda, J. B.; Venable, R. M.; Freites, J. A.; O'Connor, J. W.; Tobias, D. J.; Mondragon-Ramirez, C.; Vorobyov, I.; MacKerell, A. D., Jr.; Pastor, R. W. Update of the CHARMM All-Atom Additive Force Field for Lipids: Validation on Six Lipid Types. J. Phys. Chem. B 2010, 114, 7830-7843.

(5) Klauda, J. B., Venable, R. M., MacKerell, A. D., and Pastor, R. W. Considerations for Lipid Force Field Development. In Computational Modeling of Membrane Bilayers; 2008, 60, 1-48.

(6) Klauda, J. B.; Monje, V.; Kim, T.; Im, W. Improving the CHARMM Force Field for Polyunsaturated Fatty Acid Chains. J. Phys. Chem. B 2012, 116, 9424-9431.

(7) Lim, J. B.; Rogaski, B.; Klauda, J. B. Update of the Cholesterol Force Field Parameters in CHARMM. J. Phys. Chem. B 2012, 116, 203-210. 
(8) Jambeck, J. P. M.; Lyubartsev, A. P. Derivation and Systematic Validation of a Refined All-Atom Force Field for Phosphatidylcholine Lipids. J. Phys. Chem. B 2012, 116, 3164-3179.

(9) Jambeck, J. P. M.; Lyubartsev, A. P. An Extension and Further Validation of an All-Atomistic Force Field for Biological Membranes. J. Chem. Theory Comput. 2012, 8, 2938-2948.

(10) Monticelli, L.; Kandasamy, S. K.; Periole, X.; Larson, R. G.; Tieleman, D. P.; Marrink, S. J. The MARTINI Coarse-Grained Force Field: Extension to Proteins. J. Chem. Theory Comput. 2008, 4, 819834.

(11) Marrink, S. J.; Risselada, H. J.; Yefimov, S.; Tieleman, D. P.; de Vries, A. H. The MARTINI Force Field: Coarse Grained Model for Biomolecular Simulations. J. Phys. Chem. B 2007, 111, 7812-7824.

(12) Dickson, C. J.; Rosso, L.; Betz, R. M.; Walker, R. C.; Gould, I. R. GAFFlipid: a General Amber Force Field for the Accurate Molecular Dynamics Simulation of Phospholipid. Soft Matter 2012, 8, 96179627.

(13) Ulmschneider, J. P.; Ulmschneider, M. B. United Atom Lipid Parameters for Combination with the Optimized Potentials for Liquid Simulations All-Atom Force Field. J. Chem. Theory Comput. 2009, 5, $1803-1813$

(14) Sachs, J. N.; Petrache, H. I.; Woolf, T. B. Interpretation of Small Angle X-ray Measurements Guided by Molecular Dynamics Simulations of Lipid Bilayers. Chem. Phys. Lipids 2003, 126, 211-223.

(15) Benz, R. W.; Castro-Roman, F.; Tobias, D. J.; White, S. H. Experimental Validation of Molecular Dynamics Simulations of Lipid Bilayers: A New Approach. Biophys. J. 2005, 88, 805-817.

(16) de Vries, A. H.; Chandrasekhar, I.; van Gunsteren, W. F.; Hunenberger, P. H. Molecular Dynamics Simulations of Phospholipid Bilayers: Influence of Artificial Periodicity, System Size, and Simulation Time. J. Phys. Chem. B 2005, 109, 11643-11652.

(17) Kučerka, N.; Perlmutter, J. D.; Pan, J.; Tristram-Nagle, S.; Katsaras, J.; Sachs, J. N. The Effect of Cholesterol on Short- and LongChain Monounsaturated Lipid Bilayers as Determined by Molecular Dynamics Simulations and X-ray Scattering. Biophys. J. 2008, 95, 2792-2805.

(18) Kučerka, N.; Nagle, J. F.; Sachs, J. N.; Feller, S. E.; Pencer, J.; Jackson, A.; Katsaras, J. Lipid Bilayer Structure Determined by the Simultaneous Analysis of Neutron and X-ray Scattering Data. Biophys. J. 2008, 95, 2356-2367.

(19) Pan, J. J.; Tieleman, D. P.; Nagle, J. F.; Kučerka, N.; TristramNagle, S. Alamethicin in Lipid Bilayers: Combined Use of X-ray Scattering and MD Simulations. Biochim. Biophys. Acta, Biomembr. 2009, 1788, 1387-1397.

(20) Kučerka, N.; Katsaras, J.; Nagle, J. F. Comparing Membrane Simulations to Scattering Experiments: Introducing the SimtoExp Software. J. Membr. Biol. 2010, 235, 43-50.

(21) Braun, A. R.; Sachs, J. N. Extracting Experimental Measurables from Molecular Dynamics Simulations of Membranes. Annu. Rep. Comput. Chem. 2011, 7, 125-150.

(22) Klauda, J. B.; Kučerka, N.; Brooks, B. R.; Pastor, R. W.; Nagle, J. F. Simulation-Based Methods for Interpreting X-ray Data from Lipid Bilayers. Biophys. J. 2006, 90, 2796-2807.

(23) Nagle, J. F. Basic Quantities in Model Biomembranes. Faraday Discuss. 2013, 161, 11-29.

(24) Nagle, J. F.; Tristram-Nagle, S. Structure of Lipid Bilayers. Biochim. Biophys. Acta, Rev. Biomembr. 2000, 1469, 159-195.

(25) Berger, O.; Edholm, O.; Jahnig, F. Molecular Dynamics Simulations of a Fluid Bilayer of Dipalmitoylphosphatidylcholine at Full Hydration, Constant Pressure, and Constant Temperature. Biophys. J. 1997, 72, 2002-2013.

(26) Hess, B.; Kutzner, C.; van der Spoel, D.; Lindahl, E. GROMACS 4: Algorithms for Highly Efficient, Load-Balanced, and Scalable Molecular Simulation. J. Chem. Theory Comput. 2008, 4, 435-447.

(27) Van der Spoel, D.; Lindahl, E.; Hess, B.; Groenhof, G.; Mark, A. E.; Berendsen, H. J. C. Gromacs: Fast, Flexible, and Free. J. Comput. Chem. 2005, 26, 1701-1718.
(28) Lindahl, E.; Hess, B.; Van Der Spoel, D. GROMACS 3.0: A Package for Molecular Simulation and Trajectory Analysis. J. Mol. Model. 2001, 7, 306-317.

(29) Berendsen, H. J. C.; Vanderspoel, D.; Vandrunen, R. Gromacs a Message-Passing Parallel Molecular-Dynamics Implementation. Comput. Phys. Commun. 1995, 91, 43-56.

(30) Essmann, U.; Perera, L.; Berkowitz, M. L.; Darden, T.; Lee, H.; Pedersen, L. G. A Smooth Particle Mesh Ewald Method. J. Chem. Phys. 1995, 103, 8577-8593.

(31) Hess, B. P-LINCS: A Parallel Linear Constraint Solver for Molecular Simulation. J. Chem. Theory Comput. 2008, 4, 116-122.

(32) Miyamoto, S.; Kollman, P. A. Settle - an Analytical Version of the Shake and Rattle Algorithm for Rigid Water Models. J. Comput. Chem. 1992, 13, 952-962.

(33) Nose, S. A Molecular Dynamics Method for Simulations in the Canonical Ensemble. Mol. Phys. 1984, 255-268.

(34) Parrinello, M.; Rahman., A. Polymorphic Transitions in Single Crystals: A New Molecular Dynamics Method. J. Appl. Phys. 1981, 52, $7182-7190$.

(35) Michaud-Agrawal, N.; Denning, E. J.; Woolf, T. B.; Beckstein, O. Software News and Updates MDAnalysis: A Toolkit for the Analysis of Molecular Dynamics Simulations. J. Comput. Chem. 2011, 32, 2319-2327.

(36) Braun, A. R.; Brandt, E. G.; Edholm, O.; Nagle, J. F.; Sachs, J. N. Determination of Electron Density Profiles and Area from Simulations of Undulating Membranes. Biophys. J. 2011, 100, 2112-2120.

(37) Petrache, H. I.; Feller, S. E.; Nagle, J. F. Determination of component volumes of lipid bilayers from simulations. Biophys. J. 1997, 72, 2237-2242.

(38) Pan, J.; Tristram-Nagle, S.; Kučerka, N.; Nagle, J. F. Temperature Dependence of Structure, Bending Rigidity, and Bilayer Interactions of Dioleoylphosphatidylcholine Bilayers. Biophys. J. 2008, 94, 117-124.

(39) Pan, J. J.; Tristram-Nagle, S.; Nagle, J. F. Effect of Cholesterol on Structural and Mechanical Properties of Membranes Depends on Lipid Chain Saturation. Phys. Rev. E 2009, 80, 021931-021912.

(40) Liu, Y. F.; Nagle, J. F. Diffuse Scattering Provides Material Parameters and Electron Density Profiles of Biomembranes. Phys. Rev. E 2004, 69, 040901-040904(R).

(41) Kučerka, N.; Tristram-Nagle, S.; Nagle, J. F. Structure of Fully Hydrated Fluid Phase Lipid Bilayers with Monounsaturated Chains. J. Membr. Biol. 2005, 208, 193-202.

(42) Katsaras, J.; Kučerka, N.; Gallova, J.; Uhrikova, D.; Balgavy, P.; Bulacu, M.; Marrink, S. J. Areas of Monounsaturated Diacylphosphatidylcholines. Biophys. J. 2009, 97, 1926-1932.

(43) Nagle, J. F.; Wiener, M. C. Structure of Fully Hydrated Bilayer Dispersions. Biochim. Biophys. Acta 1988, 942, 1-10.

(44) Wiener, M. C.; Suter, R. M.; Nagle, J. F. Structure of the Fully Hydrated Gel Phase of Dipalmitoylphosphatidylcholine. Biophys. J. $1989,55,315-325$.

(45) Wiener, M. C.; White, S. H. Structure of a Fluid Dioleoylphosphatidylcholine Bilayer Determined by Joint Refinement of X-ray and Neutron-Diffraction Data 0.2. Distribution and Packing of Terminal Methyl-Groups. Biophys. J. 1992, 61, 428-433.

(46) Nagle, J. F.; Wiener, M. C. Relations for Lipid Bilayers Connection of Electron-Density Profiles to Other Structural Quantities. Biophys. J. 1989, 55, 309-313.

(47) Rawicz, W.; Olbrich, K. C.; McIntosh, T.; Needham, D.; Evans, E. Effect of Chain Length and Unsaturation on Elasticity of Lipid Bilayers. Biophys. J. 2000, 79, 328-339.

(48) Raghunathan, M.; Zubovski, Y.; Venable, R. M.; Pastor, R. W.; Nagle, J. F.; Tristram-Nagle, S. Structure and Elasticity of Lipid Membranes with Genistein and Daidzein Bioflavinoids Using X-ray Scattering and MD Simulations. J. Phys. Chem. B 2012, 116, 39183927.

(49) Waheed, Q.; Edholm, O. Undulation Contributions to the Area Compressibility in Lipid Bilayer Simulations. Biophys. J. 2009, 97, 2754-2760. 
(50) Brandt, E. G.; Braun, A. R.; Sachs, J. N.; Nagle, J. F.; Edholm, O. Interpretation of Fluctuation Spectra in Lipid Bilayer Simulations. Biophys. J. 2011, 100, 2104-2111.

(51) Evans, E.; Rawicz, W.; Smith, B. A. Back to the Future: Mechanics and Thermodynamics of Lipid Biomembranes. Faraday Discuss. 2013, 161, 591-611.

(52) Tristram-Nagle, S.; Petrache, H. I.; Nagle, J. F. Structure and Interactions of Fully Hydrated Dioleoylphosphatidylcholine Bilayers. Biophys. J. 1998, 75, 917-925. 